DOI $10.31489 / 2020$ No2/6-10

UDC 538.9

\title{
FORMATION OF MAGNETIC PROPERTIES OF FERRITES DURING RADIATION-THERMAL SINTERING
}

\author{
Stary O'., Malyshev A.V. ${ }^{2}$, Lysenko E.N. ${ }^{2}$, Petrova A. ${ }^{2}$ \\ ${ }^{1}$ Czech Technical University in Prague, Prague, Czech Republic \\ 2Tomsk Polytechnic University, 30 Lenin Avenue, 634050 Tomsk, Russia, ‘malyshev@tpu.ru
}

\begin{abstract}
The results of a comparative analysis of the laws governing the formation of ferrite hysteresis loop parameters sintered in thermal and radiation-thermal conditions were shown. The influence of radiation exposure on the interconversion of microstructure defects and their content in ferrites, depending on the duration and temperature of treatment, was established. Also, it was shown that recrystallization grain growth under irradiation conditions is ahead of grain growth during thermal heating. The observed radiation effects were associated with the effect of radiation on the microstructure. The magnetic parameters are uniquely determined by the compaction of the sample.
\end{abstract}

Keywords: effects of radiation, ferrites, magnetic properties, microstructure, sintering, hysteresis loop, coercive force, pore volume.

\section{Introduction}

The most universal requirement for the microstructure of ferrites is the condition for the maximum density of the material and the equigranular structure [1,2]. For comply with these requirements, at economically reasonable firing times, several methods have been developed to increase the activity of sintering processes. This is the two-stage introduction of the components, the addition of ferrite with a ferrite powder of the same composition, the presence of a liquid phase, forced sintering, the application of ultrasound to the sintering process $[1,3,4-12]$.

In recent years, the effects of ionizing radiation fluxes in the preparation and modification of materials were developed. In this case, the fundamental phenomenon of multiple increases in the synthesis of multicomponent powder materials [13-15] and sintering [16-20] under radiation-thermal conditions was discovered. The sintering processes of lithium titanium ferrites were most fully studied under such specific conditions of the combined action of high temperatures and intense electron flows [21, 22]. The patterns of compaction of ferrite compacts were established and a multiple increase in the compaction rate of lithiumtitanium ferrite compacts under such sintering conditions was shown [23, 24]. Moreover, almost all studies concerned the study of the structural, phase, and mechanical properties of the materials thus obtained, i.e. concerned such areas of science as sintering physics and powder metallurgy.

However, the main functional characteristics of ferrimagnets are their magnetic properties, and the ultimate goal of any ferrite production technology is to achieve a given level of operational properties. Therefore, control over the formation of the basic electromagnetic parameters is a prerequisite in the development of new technological processes. On the other side, the processes of magnetization reversal and electric transport in ferrites are closely related to the features of their microstructure. Therefore, the regularities of change in electromagnetic characteristics can be used as a source of important information about the nature of the processes occurring during sintering and, therefore, can help in a more in-depth interpretation of the mechanisms of radiation-thermal sintering.

\section{Object of study and experimental technique}

In the present work powders of lithium-titanium ferrite synthesized from a mechanical mixture of oxides and carbonates containing (wt.\%) were used: $\mathrm{Li}_{2} \mathrm{CO}_{3}-11.2 ; \mathrm{TiO}_{2}-18.65 ; \mathrm{ZnO}-7.6 ; \mathrm{MnCO}_{3}-2.74$; the rest is $\mathrm{Fe}_{2} \mathrm{O}_{3}$. For prepare a press powder, a 10\% solution of polyvinyl alcohol in an amount of $12 \mathrm{wt}$. \% of charge. Press samples in the form of tablets and ring cores with a thickness of $2 \mathrm{~mm}$ are made by cold unilateral pressing. The optimum pressing pressure is selected experimentally. It was found that at pressing pressures below $40 \mathrm{MPa}$, the press samples are characterized by a low density, and at pressing pressures of 
more than $250 \mathrm{MPa}$, the samples have cracks and delaminations (the phenomenon of repressing). Thus, the most optimal pressing pressure, which provides an acceptable density of both raw and sintered samples, is in the range (110-200) MPa.

The following pressing mode was used in the work: $\mathrm{P}=130 \mathrm{MPa}$, the exposure time of the material under pressure $1 \mathrm{~min}$; and two modes of sintering of press samples: radiation-thermal (RT) and thermal (T). RT-sintering was carried out by irradiating the samples with a pulsed electron beam with an energy of the (1.5-2.0) MeV using the ILU-6 accelerator. The beam current in the pulse was (0.5-0.9) A, the irradiation pulse duration was $500 \mu \mathrm{s}$, the pulse repetition rate was $(5-50) \mathrm{Hz}$, and the work piece heating rate was $1000{ }^{\circ} \mathrm{C} / \mathrm{min}$. The samples were irradiated in a box of lightweight fireclay with a bottom thickness of $15 \mathrm{~mm}$. On the irradiation side, the box was covered with a radiation-transparent tread of mass thickness $0.1 \mathrm{~g} \mathrm{~cm}^{-1}$. The temperature was measured by a control sample placed close to the sintered blanks.

Sintering in thermal furnaces (T-sintering) was carried out in a preheated chamber electric furnace, which ensured a heating rate comparable with the rate of radiation heating. The cell design and temperature control technique are similar to those used in RT-sintering. Both sintering modes were carried out in air. The magnetic characteristics of ferrites were measured by a standard method with an F 5063 ferrometer. The magnitude of the magnetizing field was 5 Oersted (Oe) at a field frequency of $50 \mathrm{~Hz}$. The degree of porosity and grain size were studied on polished, polished and etched sections of sintered samples using an MBI15Us optical microscope. The kinetic dependencies of the hysteresis loop parameters $B_{m}, B_{r} / B_{m}$ and $H_{c}$ of ferrite samples calcined in the temperature range (1173-1373) K were studied.

\section{Results and discussion}

Figure 1 shows the kinetic dependencies of the saturation magnetic induction $B_{m}$ for samples with a sintering temperature of $1173,1273,1373 \mathrm{~K}$. It can be seen that the $B_{m}$ increases with increasing duration and sintering temperature. Under conditions of radiation-thermal sintering, the absolute values of $B_{m}$ exceed the data obtained by thermal sintering. The maximum difference falls on the lower boundary of the sintering temperature range: with increasing temperature, the effect of activating the change in $B_{m}$ decreases.

The saturation magnetic induction is determined by the crystal chemistry of the material and the relative magnitude of nonmagnetic phases, including pores [25]. The influence of non-magnetic phases is associated with the appearance of demagnetizing fields in their vicinity, and also, these phases do not contribute to the magnetization of ferrite. The initial state of the sample can be considered as a set of magnetic particles separated by air layers, i.e., as a magnetodielectric. This state is characterized by a large internal demagnetizing factor $N$. It is also significant that the saturation magnetization of a magnetodielectric is proportional not only to the saturation magnetization of particles but also to the volumetric filling coefficient $P / \bar{J}_{S}=P \hat{f}_{S}$ [6]. Therefore, the resulting saturation induction of the billet is characterized by small $B_{m}$. According to Figure 1, it can be seen that the rearrangement of particles (reduction of the air gap) occurs both at the stage of heating and in the isothermal sintering mode. Exposure to radiation also intensifies particle rapprochement. It is characteristic that at the nonisothermal stage of sintering, the radiation effect increases with temperature, and at the isothermal stage, it decreases. This can be seen from the transition from the asymmetric curves $l$ and $l^{\prime}$ (at $\mathrm{T}=1173 \mathrm{~K}$ ) to the almost symbate curves 3 and $3^{\prime}$ (at $\mathrm{T}=1373 \mathrm{~K}$ ). The initial values of $B_{m}($ at $\tau=0)$, on the contrary, differ more from one another with increasing temperature. Obviously, the polar differences in the temperature dependence of the radiation effect during heating and at the isothermal sintering stage indicate a difference in the mechanisms of particle compaction at these stages.

Figure 2 shows the kinetic curves of the variation of the coefficient of squareness $\gamma=B_{r} / B_{m}$ in the temperature range (1173-1373) K. The existence of the time dependence of $\gamma$ indicates that during sintering, partial elimination of defects that are the sources of magnetization reversal nuclei with a critical magnetic field strength $\mathrm{Hni}<0$. Indeed, if there are nuclei for which the critical strength of the magnetic field is caused not only by rotations of elementary magnetic moments in the direction of the axis of easy magnetization but also an induction created by reverse magnetic domains. Therefore, elimination of defects sources of reverse magnetization domains during heat treatment - will increase $\mathrm{Br}$ and, accordingly, increase $\gamma . H_{n i}<0$, then the difference between the saturation induction $B_{m}$ and the residual induction $B_{r}$.

Of all the variety of sources of reverse magnetization domains fired under the conditions of our experiment, the most probable are inclusions of side phases - intermediate synthesis products or unreacted oxides. This is supported by a sign of the diffusion nature of firing, which consists in reducing the effect of radiation stimulation of the process with an increase in sintering temperature (see Fig. 2). 


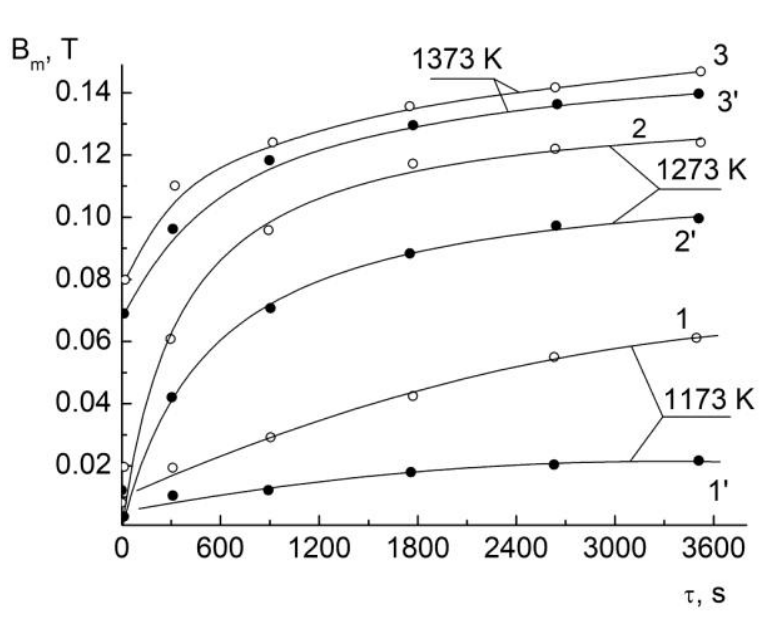

Fig.1. Dependence of saturation induction on time and sintering temperature of ferrite:

- - T-sintering; $\square$ - RT-sintering.

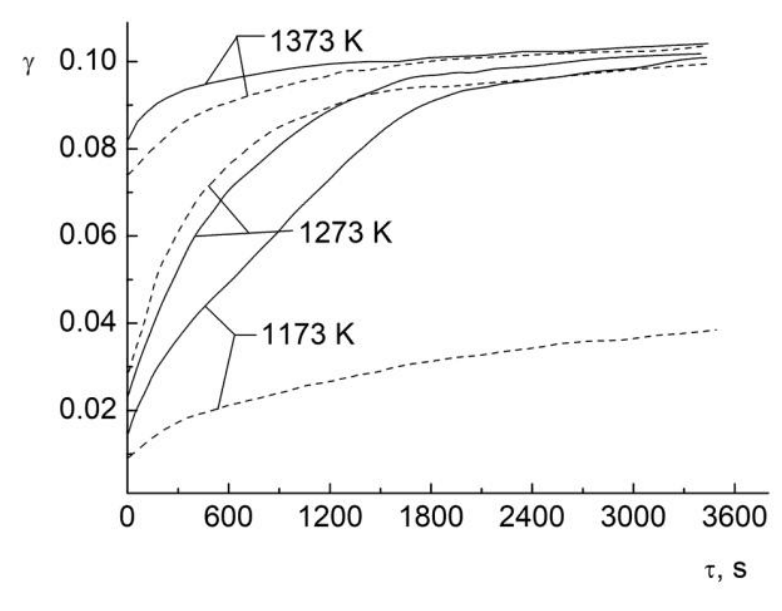

Fig.2. Dependence of coefficient of squareness of the hysteresis loop $(\gamma)$ on time and sintering temperature of ferrite: solid line - RT-sintering; dash line - Tsintering.

The radiation effect of high-temperature self-diffusion in metals behaves in a similar way, which we established under irradiation conditions identical to our experiment. Figure 3 shows the dependencies of the change in the coercive force $H_{c}$ on the duration of sintering in radiation-thermal and thermal regimes. At $T=$ $1173 \mathrm{~K}$, the $H_{c}$ monotonically increases in the entire studied sintering time interval. At $T=1273 \mathrm{~K}$, an increase in $H_{c}$ at the initial stage of sintering becomes a decreasing dependence. The maximum time dependence of $H_{c}$ in the conditions of our experiment falls on the sintering duration (12-17 $\left.\mathrm{min}\right)$. At $T=$ $1373 \mathrm{~K}$, only a decreasing dependence of $H_{c}$ on sintering time is observed. According to existing ideas, the coercive force $H_{c}$ is determined by averaging the braking forces arising from material defects. The greatest resistance to displacements of domain walls is exerted by grain boundaries (Globus theory [26-28]), as well as intragranular linear, planar, and volume inclusions (Kersten theory [29, 30], Neel [31, 32]).

At T $=1173 \mathrm{~K}$, an increase in $H_{c}$ is obviously due to a decrease in the internal demagnetizing factor $N$ due to compaction of the billet (with a corresponding increase in the effective magnetic field). As in the case of $B_{m}$, the effect of radiation effects of compaction is clearly manifested at this temperature. At $1273 \mathrm{~K}$, an increase in $H_{c}$ at the initial stage of sintering is also due to compaction of the billet. However, upon reaching a sufficient degree of compaction, changes in the intensity of the internal magnetic field no longer play a noticeable role. From this moment in time, the changes in $B_{m}$ are also relatively small (Fig. 1). A subsequent decrease in $H_{c}$ can be associated with a recrystallization increase in grain size and a decrease in the volume fraction of inclusions. At this stage, radiation effects are also clearly manifested. At $\mathrm{T}=1373 \mathrm{~K}$, the necessary degree of compaction is achieved in the process of heating the billet for sintering. Therefore, in the isothermal mode, only a decrease in $H_{c}$ is observed due to the above reasons.

It is characteristic that the radiation effects, depending on $H_{c}$ on the sintering duration at $1373 \mathrm{~K}$, are not explicitly expressed. At the same time, recrystallization grain growth under irradiation conditions is ahead of grain growth during thermal heating. On this basis, the healing process of intragranular defect can be considered a more probable reason for the decrease in $H_{c}$. This process, obviously, has a diffusion character. But, as discussed above, with increasing temperature, the intensity of diffusion processes weakly depends on the effect of radiation. This fact is consistent with the assumptions made.

In discussing the above results, we ignored the possible direct effect of radiation on the formation of the magnetic structure (for example, due to the effect on the cation distribution). The observed radiation effects were associated with the effect of radiation on the microstructure. The validity of this approach is proved by the data presented in Fig. 4, Fig. 5 and Fig. 6, where the dependencies of the magnetic parameters on the pore volume are plotted. The parameters were taken from the data in Fig. 1, Fig. 2 and Fig. 3. The results are depicted in Fig. 4, Fig. 5 and Fig. 6 show that the all parameters are uniquely determined by the compaction of the ferrite samples. It also follows from the presented results that the role of stimulating factors (temperature and radiation) in accelerating of sintering process is reduced. 


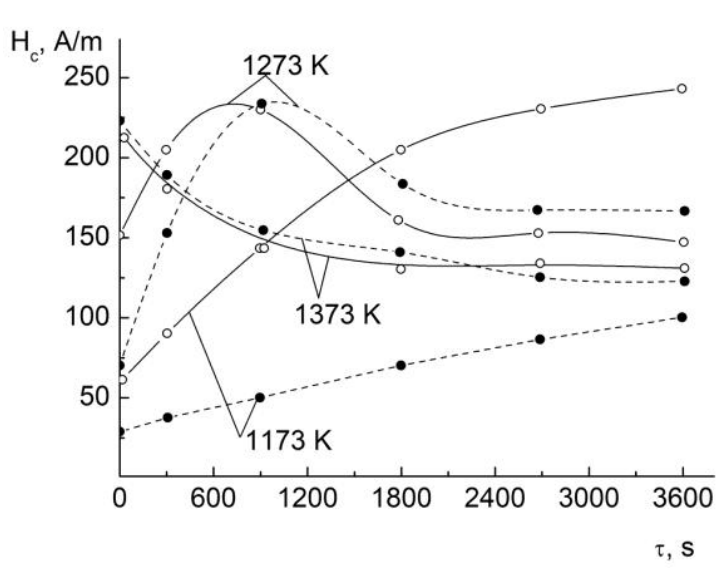

Fig.3. Dependence of coercive force on time and sintering temperature of ferrite: $\bullet-\mathrm{T}$-sintering; $\mathrm{O}-$ RT-sintering.

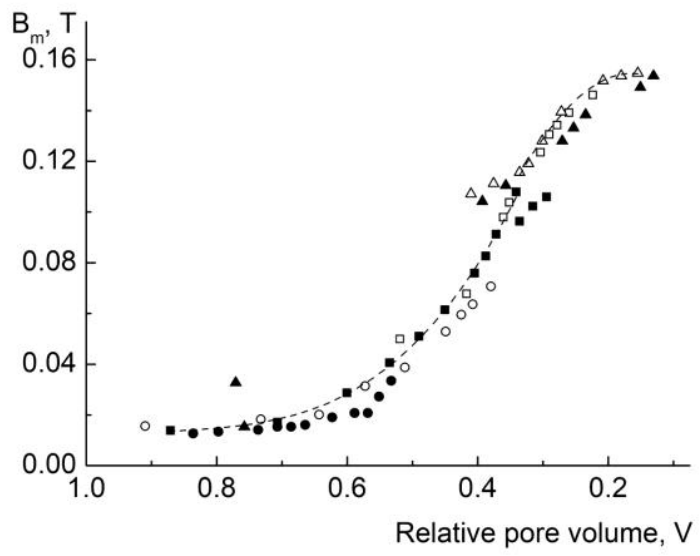

Fig.5. Dependence of saturation induction on relative pore volume of ferrites sintered at various temperatures: $\bullet$, O - $1173 \mathrm{~K} ; \boldsymbol{\square}, \square-1273 \mathrm{~K} ; \boldsymbol{\Delta}, \Delta-1373 \mathrm{~K}$.

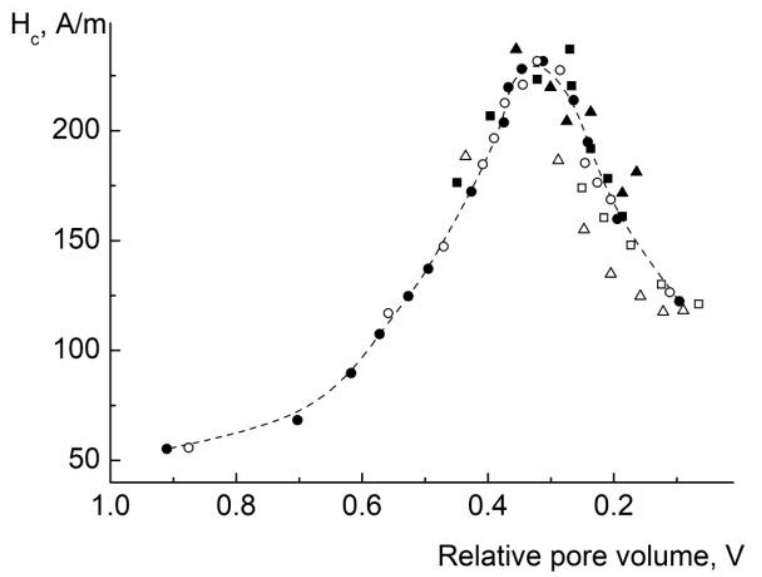

Fig.4. Dependence of coercive force on relative pore volume: •, O-1173K; $\mathbf{\square}, \square-273 \mathrm{~K} ; \boldsymbol{\Delta}, \Delta-1373 \mathrm{~K}$.

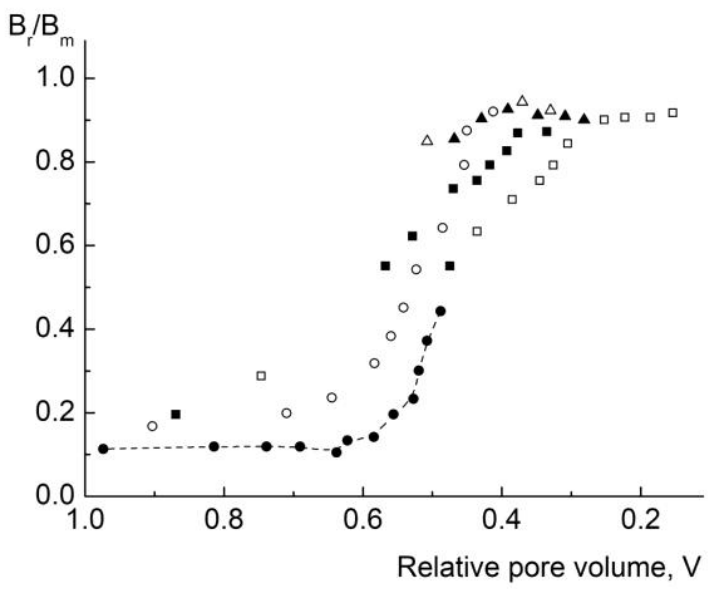

Fig.6. Dependence of coefficient of squareness of the hysteresis loop on relative pore volume of samples sintered at various temperatures:

$\bullet, \bigcirc-1173 \mathrm{~K} ; \mathbf{\square}, \square-1273 \mathrm{~K} ; \boldsymbol{\Delta}, \Delta-1373 \mathrm{~K}$.

\section{Conclusion}

This investigation presents the results of a study of kinetic laws of formation of the parameters of the hysteresis loop for ferrites sintered under thermal and radiation-thermal conditions. The scientific novelty of the study is to determine the effect of radiation exposure on the mutual conversion of microstructure defects and their content in ferrites, depending on the duration and temperature of treatment. The authors showed for the first time that recrystallization grain growth under irradiation conditions is ahead of grain growth during thermal heating. The observed radiation effects were associated with the effect of radiation on the ferrite microstructure. In addition, it is reliably established that all magnetic parameters are uniquely determined by the compaction of ferrite samples.

\section{Acknowledgments}

The research is funded by The Ministry of Education and Science of the Russian Federation as part of the "Science" Program (project FSWW-2020-0014). The results of the coercive force of ferrite samples were obtained within the RFBR grant (project No. 20-07-00662). The experimental calculations are carried out at Tomsk Polytechnic University within the framework of Tomsk Polytechnic University Competitiveness Enhancement Program grant. 


\section{REFERENCES}

1 Letyuk L.M., Nifontov V.A., Babich E.A., Gorelik S.S. Effect of low-melting additives on the formation of the microstructure and the properties of ferrites with a rectangular hysteresis loop. Izv. Akad. Nauk Neorg. Mater. 1976, Vol.12, pp. 2023 - 2026.

2 Zhuravlev G.I., Golubkov L.A., Strakhova T.A. Basic types of microstructure of ferrites and means of obtaining them. Soviet Powder Metallurgy and Metal Ceramics. 1990, Vol.29, pp. 478 - 480.

3 Dmitriev M.V., Letyuk L.M., Shipko M.N. Study of oxygen diffusion in the surface layers of Mn-Zn ferrites. Technical physics. 1982, Vol.27, pp. $338-339$.

4 Letyuk L.M. Recrystallization of ferrites and its effect on the processes of microstructure formation in ferrospinels. Soviet Powder Metallurgy and Metal Ceramics. 1980, Vol.19, No.5, pp. $359-364$.

5 Zinovik M.A., Zinovik E.V. Ferrites with rectangular and square hysteresis loops. Powder Metallurgy and Metal Ceramics. 2005, Vol.44, p. $66-74$.

6 Letyuk L.M., et al. Special features of the formation of the microstructure of ferrites sintered in the presence of a liquid phase. Izv.Vysshikh Uchebnykh Zavedenij. Chernaya Metallurgiya. 1979, Vol.11, pp. 124 - 127.

7 Micheli A.L. Preparation of lithium ferrites by coprecipitation. IEEE Transactions on Magnetics. 1970, Vol.6, pp. $606-608$.

8 Bronshteyn I.M., Dyubua B.Ch., Karasik B.S., Khinich I.I. Secondary electron emission of a platinum-barium alloy made by evaporation. Radio Eng. Electron Phys. 1974, Vol.19, pp. 108 - 111.

9 Minin V.M. Effect of sintering conditions on the microstructure and electromagnetic properties of $\mathrm{Li}-\mathrm{Mg}-\mathrm{Mn}$ ferrite memory elements. Soviet Powder Metallurgy and Metal Ceramics. 1982, Vol.21, pp. 698 - 701.

10 Zahir R., Chowdhury F.-U.-Z., Uddin M.M., et al. Structural, magnetic and electrical characterization of Cdsubstituted Mg ferrites synthesized by double sintering technique. J. Magn. Magn. Mater. 2016, Vol. 410 , pp. 55 - 62.

11 Manjura Hoque S., Abdul Hakim M., Mamun Al, et al. Study of the bulk magnetic and electrical properties of $\mathrm{MgFe}_{2} \mathrm{O}_{4}$ synthesized by chemical method. Materials Sciences and Applications. 2011, Vol.2, pp. 1564 - 1569.

$12 \mathrm{Hu}$ J., Yan M., Luo W., Wu J.M. Effects of microstructure on the temperature dependence of relative initial permeability of NiCuZn ferrites. Physica B. 2007, Vol.400, pp. $119-123$.

13 Surzhikov A.P., Pritulov A.M., Lysenko E.N., et al. Calorimetric investigation of radiation-thermal synthesized lithium pentaferrite. Journal of Thermal Analysis and Calorimetry. 2010, Vol. 101, No. 1, pp. 11-13.

14 Yurov V.M., Baltabekov A.S., Laurinas V.C., Guchenko S.A. Dimensional effects and surface energy of ferroelectric crystals. Eurasian Physical Technical Journal. 2019, Vol.16, No.1, pp. 18 - 23.

15 Surzhikov A.P., Frangulyan T.S., Ghyngazov S.A., et al. Pysics of magnetic phenomena: Investigation of electroconductivity of lithium pentaferrite. Russian Physics Journal. 2006, Vol. 49, No. 5, pp. 506-510.

16 El-Shobaky G.A., Ibrahim A.A. Solid-solid interactions between ferric oxide and lithium carbonate and the thermal stability of the lithium ferrites produced. Thermochim. Acta. 1987, Vol.118, pp. $151-158$.

17 Salimov R.A., Cherepkov V.G., Golubenko J.I., et al. D.C. high power electron accelerators of ELV-series: status, development, applications. J. Radiation Phys. Chem. 2000, Vol.57, pp. $661-665$.

18 Cleland M.R., Parks L.A. Medium and high-energy electron beam radiation processing equipment for commercial applications. Nucl. Instr. Meth. B. 2003, Vol.208, pp. 74 - 89.

19 Mehnert R. Review of industrial applications of electron accelerators. Nucl.Instr.Meth. 1996, Vol.113, pp.81-87.

20 Neronov V.A., Voronin A.P., Tatarintseva M.I., Melekhova T.E., Auslender V.L. Sintering under a high-power electron beam. J. Less-Common Metals. 1986, Vol.117, pp. 391 - 394.

21 Surzhikov A.P., Peshev V.V., Pritulov A.M., Gyngazov S.A. Grain-boundary diffusion of oxygen in polycrystalline ferrites. Russian Physics Journal. 1999, Vol. 42, No. 5, pp. 490-495.

22 Boldyrev V.V., Voronin A.P., Gribkov O.S., Tkachenko E.V., Karagedov G.R., Yakobson B.I., Auslender V.L. Radiation-thermal synthesis. Current achievement and outlook. J. Solid State Ion. 1989, Vol.36, pp. 1 - 6.

23 Surzhikov A.P., Lysenko E.N., Malyshev A.V., et al. Study of the Radio-Wave Absorbing Properties of a Lithium-Zinc Ferrite Based Composite. Russian Physics Journal. 2014, Vol. 57, No. 5, pp. 621-626.

24 Surzhikov A.P., et al. Structural, electromagnetic, and dielectric properties of lithium-zinc ferrite ceramics sintered by pulsed electron beam heating. Ceramics International. 2017, Vol. 43, No. 13, pp. 9778-9782.

25 Smith J., Wijn H.P.J. Ferrites: Physical properties of ferromagnetic oxides in relation to their technical application. 1959, Eindhoven, Phillips Technical Library, 233p.

26 Globus A. Influence des dimensions des parois la permeability. C. R. Acad. Sci. 1962, Vol.255, pp. 17091711. [in French]

27 Globus A., Guyot M. Wall displacement and bulging in magnetization mechanisms of the hysteresis loop. Phys. Status Solidi B. 1972, Vol.52, pp. $427-431$.

28 Globus A. Some physical consideration about the domain wall sine. Theory of magnetization mechanisms, J.Phys. (France). 1977, Vol.38, pp. $1-15$.

29 Kersten M. Zur wirkung der versetzungen auf die anfangspermeabilität von nickel im rekristallisierten und im plastisch verformten. Zustand Annalen der Physik. 1957, Vol.20, No.1-6, pp. 337 - 344. [in German]

30 Kersten M. Reversible und irreversible magnetisierun-gsanderungerlangs der hystereschleife, Z. Angew. Phys. 1955, Vol.7, pp. 397 - 407. [in German]

31 Neel L. Energie magnetocrictalline d'ur macrocristal subdivise on crystallites guadretigues, Compt. Rend. Acad. Sci. 1963, Vol.257, pp. 2917 - 2921.

32 Neel L. Defaults ponetuels dansles solides ferromagnetiques et ordre directional. J. Phys. (France). 1963, Vol.24, pp. $513-516$. 